\title{
On a Generalized Demosaicking Procedure: A Taxonomy of Single-Sensor Imaging Solutions
}

\author{
Rastislav Lukac and Konstantinos N. Plataniotis \\ The Edward S. Rogers Sr. Dept. of Electrical and Computer Engineering, \\ University of Toronto, 10 King's College Road, Toronto, M5S 3G4, Canada \\ \{lukacr, kostas\}@dsp.utoronto.ca \\ http://www.dsp.utoronto.ca/ lukacr
}

\begin{abstract}
This paper presents a generalized demosaicking procedure suitable for single-sensor imaging devices. By employing an edge-sensing mechanism and a spectral model, the proposed demosaicking framework preserves both the spatial and spectral characteristics of the captured image. Experimental results reported in this paper indicate that the solutions designed within the proposed framework produce visually pleasing full color, demosaicked images.
\end{abstract}

\section{Introduction}

Color filter array (CFA) interpolation or demosaicking is an integral step in single-sensor imaging solutions such as digital cameras, image-enabled wireless phones, and visual sensors for surveillance and automotive applications, [1]-[6]. The CFA is used to separate incoming light into a mosaic of the color components (Fig.1a). The sensor, usually a charge-coupled device (CCD) or complementary metal oxide semiconductor (CMOS) sensor, is essentially a monochromatic device [1],[7], and thus, the raw data that acquires in conjunction with the CFA constitute a $K_{1} \times K_{2}$ gray-scale image $z$ with scalar pixels $z_{(p, q)}$, with $p=1,2, \ldots, K_{1}$ and $q=1,2, \ldots, K_{2}$ denoting the image row and column, respectively. The two missing color components are estimated from the adjacent pixels using the demosaicking process to produce the full-color demosaicked image [8]-[11].

Although a number of CFA have been proposed, the three-color Red-GreenBlue (RGB) Bayer CFA pattern (Fig.1a) [12] is the most commonly used due to the simplicity of the subsequent demosaicking procedure. Assuming the GRGR phase in the first row, a Bayer CFA image $z$, depicted in Fig.2a, can be transformed to a $K_{1} \times K_{2}$ three-channel image $\mathbf{x}$ (Fig.2b) as follows [1],[13]:

$$
\mathbf{x}_{(p, q)}=\left\{\begin{array}{l}
{\left[z_{(p, q)}, 0,0\right] \text { for } p \text { odd and } q \text { even }} \\
{\left[0,0, z_{(p, q)}\right] \text { for } p \text { even and } q \text { odd }} \\
{\left[0, z_{(p, q)}, 0\right] \text { otherwise. }}
\end{array}\right.
$$

where $\mathbf{x}_{(p, q)}=\left[x_{(p, q) 1}, x_{(p, q) 2}, x_{(p, q) 3}\right]$ denotes the color vector. The values $x_{(p, q) k}$ indicate the $\mathrm{R}(k=1), \mathrm{G}(k=2)$, or $\mathrm{B}(k=3)$ CFA components. Since the sensor image $z$ is a mosaic-like gray-scale image, the missing components in $\mathbf{x}_{(p, q)}$ are set equal to zero to indicate their portion to the coloration of $\mathbf{x}$. 


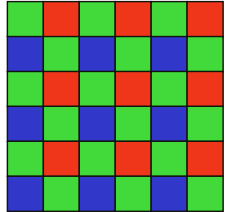

(a)

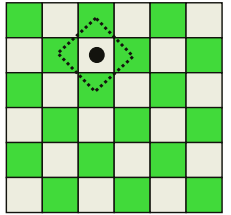

(b)

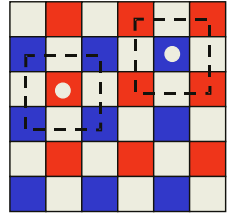

(c)

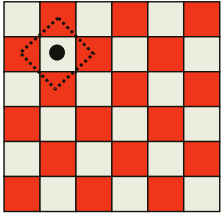

(d)

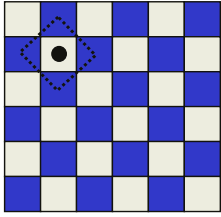

(e)

Fig. 1. (a) Bayer CFA pattern with the GRGR phase in the first row, (b-e) spatial arrangements of the four-neighboring color components observed during the proposed demosaicking procedure: (b,d,e) $\zeta=\{(p-1, q),(p, q-1),(p, q+1),(p+1, q)\},(\mathrm{c})$ $\zeta=\{(p-1, q-1),(p-1, q+1),(p+1, q-1),(p+1, q+1)\}$

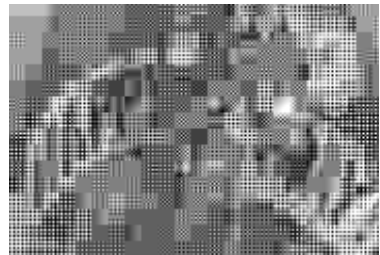

(a)

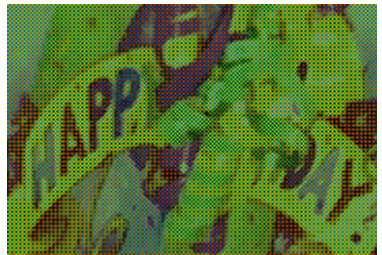

(b)

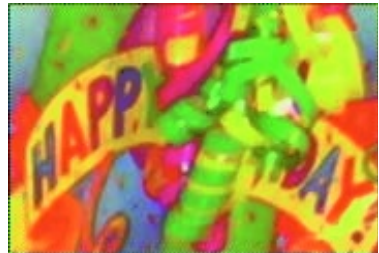

(c)

Fig. 2. Single-sensor imaging: (a) a gray-scale Bayer CFA sensor image, (b) a Bayer CFA image arranged as a color image, (c) a full-color, demosaicked image

\section{A Generalized Demosaicking Procedure}

Due to the dominance of the G component in the Bayer CFA pattern, most demosaicking procedures, for example those listed in [1]-[4],[7]-[9], start the process by interpolating the $\mathrm{G}$ color plane. In order to quantify the contribution of the adjacent samples, the missing component $x_{(p, q) k}$ is calculated as follows:

$$
x_{(p, q) k}=\sum_{(i, j) \in \zeta}\left\{w_{(i, j)}^{\prime} x_{(i, j) k}\right\}
$$

where $x_{(i, j) k}$ denotes the $k$-th components of the color vector $\mathbf{x}_{(i, j)}=\left[x_{(i, j) 1}\right.$, $\left.x_{(i, j) 2}, x_{(i, j) 3}\right]$, with $(i, j) \in \zeta$ denoting the spatial location arrangements on the image lattice (Figs.1b-e).

The normalized weighting coefficients $w_{(i, j)}^{\prime}$ used in (2) are defined as

$$
w_{(i, j)}^{\prime}=w_{(i, j)} / \sum_{(i, j) \in \zeta} w_{(i, j)}
$$

where $w_{(i, j)} \geq 0$ is the so-called edge-sensing weight. The weights $w_{(i, j)}$ are used to regulate the contribution of the available color components inside the spatial arrangements shown in Figs.1b-e. To ensure that the demosaicking procedure is an unbiased solution, the condition $\sum_{(i, j) \in \zeta} w_{(i, j)}^{\prime}=1$ must be satisfied, [1]. 


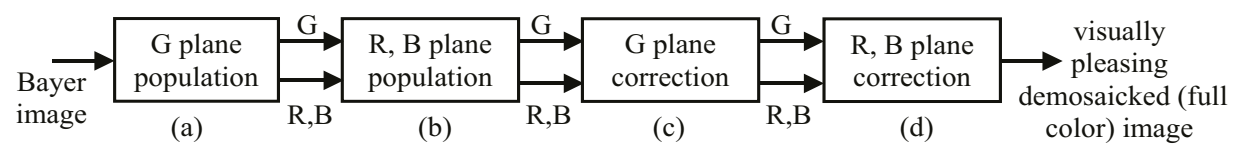

Fig. 3. Block scheme diagram of a generalized demosaicking procedure: (a,b) mandatory steps, (c,d) recommended (optional) steps

By populating the $\mathrm{G}$ color plane in Fig. $3 a$ via (2) with $k=2$ and $\zeta=$ $\{(p-1, q),(p, q-1),(p, q+1),(p+1, q)\}$ (Fig.1b), the missing R (or B) components of $\mathbf{x}$ can be obtained through the use of the spectral correlation that exists between the $\mathrm{G}$ and $\mathrm{R}$ (or $\mathrm{B}$ ) components of a natural image. Adopting the notation and concept introduced in [13], the $\mathrm{R}(k=1)$ or $\mathrm{B}(k=3)$ components $x_{(p, q) k}$ are calculated in Fig. $3 b$ as follows:

$$
x_{(p, q) k}=x_{(p, q) 2} \bar{\oplus} \sum_{(i, j) \in \zeta}\left\{w_{(i, j)}^{\prime}\left(x_{(i, j) k} \oplus x_{(i, j) 2}\right)\right\}
$$

where $\oplus$ and $\bar{\oplus}$ denote the spectral quantity formation and normalization operations, respectively. The procedure first produces the $\mathrm{R}$ and $\mathrm{B}$ components $x_{(p, q) k}$ located in the center of the shape-masks $\zeta=\{(p-1, q-1),(p-1, q+$ $1),(p+1, q-1),(p+1, q+1)\}$ (Fig.1c), and then those located in the center of the shape-masks $\zeta=\{(p-1, q),(p, q-1),(p, q+1),(p+1, q)\}$ observed for the updated planes (Figs.1d,e).

Since the G color plane was populated without the utilization of the essential spectral characteristics, the demosaicked G components obtained using (2) should be re-evaluated in Fig.3c as follows [13]:

$$
x_{(p, q) 2}=x_{(p, q) k} \bar{\oplus} \sum_{(i, j) \in \zeta}\left\{w_{(i, j)}^{\prime}\left(x_{(i, j) 2} \oplus x_{(i, j) k}\right)\right\}
$$

where $\zeta=\{(p-1, q),(p, q-1),(p, q+1),(p+1, q)\}$, as shown in Fig.1b.

Finally, the proposed demosaicking procedure completes by correcting the demosaicked $\mathrm{R}$ and $\mathrm{B}$ components (Fig.3d). This demosaicking step is realized using (4) with $k=1$ for $\mathrm{R}$ and $k=3$ for B components. As before, the spatial arrangements of the adjacent samples are described using $\zeta=\{(p-1, q-1),(p-$ $1, q+1),(p+1, q-1),(p+1, q+1)\}$ (Fig. $1 c)$ and $\zeta=\{(p-1, q),(p, q-1),(p, q+$ $1),(p+1, q)\}$ (Figs.1d,e).

\section{Taxonomy of Demosaicking Solutions}

Within the proposed generalized demosaicking framework, numerous demosaicking solutions may be constructed by changing the form of the spectral model, as well as the way the edge-sensing weights are calculated. The choice of these two construction elements essentially determines the characteristics and the performance of the single-sensor imaging solution, [1],[13],[14]. 


\subsection{Non-adaptive Versus Adaptive Solutions}

Based on the nature of the determination of $w_{(i, j)}$ in $(3)$, the demosaicking solutions can be differentiated as i) non-adaptive, and ii) adaptive demosaicking schemes.

Non-adaptive demosaicking schemes such as those listed in [15]-[18] use a simple linear averaging operator (fixed weights $w_{(i, j)}=1$ ) without considering any form of adaptive weighting, [1],[13]. Since non-adaptive schemes do not utilize structural information of the captured image to direct the demosaicking process, they produce the full-color images with blurred edges and fine details.

To restore the demosaicked image in a sharp form, adaptive demosaicking solutions use the edge-sensing weights $w_{(i, j)}$ to emphasize inputs which are not positioned across an edge and to direct the demosaicking process along the natural edges in the captured image, [1],[19],[20]. In most available designs, such as those listed in [13],[14],[21]-[25], the edge-sensing coefficients $w_{(i, j)}$ use some form of inverse gradients. In order to design a cost-effective and robust solution, the following form of $w_{(i, j)}$ defined using inverse gradients $[13],[26]$ is used throughout the paper:

$$
w_{(i, j)}=\left\{1+\sum_{(g, h) \in \varsigma}\left|x_{(i, j) k}-x_{(g, h) k}\right|\right\}^{-1}
$$

\subsection{Component-Wise Versus Spectral Model-Based Solutions}

Based on the use of the essential spectral characteristics of a captured image in the demosaicking process, the demosaicking schemes can be divided into the following two classes: i) component-wise, and ii) spectral model based solutions.

The component-wise processing solutions do not use the spectral correlation that exists between the color channels in a natural image. Such a demosaicking procedure uses $(2)$ to fully populate $\mathrm{R}(k=1), \mathrm{G}(k=2)$, and $\mathrm{B}(k=3)$ color planes. It has been widely observed [1],[20]-[28] that the omission of the spectral information in the component-wise demosaicking process in [3],[16],[17] leads to a restored output which contains color artifacts and color moire noise.

The use of the spectral model preserves the spectral correlation that exists between the color components. Since natural RGB images exhibit strong spectral correlation characteristics $[1],[6],[18]$, both researchers and practitioners in the camera image processing community rely on spectral models to eliminate spectral artifacts and color shifts. A commonality of the currently used spectral models of $[1],[15],[27],[28]$ is that they incorporate RG or BG spectral characteristics into the demosaicking process. The spectral model based demosaicking procedure, such as those used in [2],[18],[22],[23],[25], first populates the G color plane (Fig.3a) via (2), and then use the spectral characteristics in the demosaicking steps (Figs.3b-d) defined via (4), (5). It has been shown in [13] that the use of $\oplus$ and $\bar{\oplus}$ in (4)-(5) generalize the previous spectral models. Assuming for the simplicity the color-difference based modelling concept, the spectral modelling operators $\oplus$ and $\bar{\oplus}$ denote the addition and subtraction operations, respectively, and these modelling operations are used throughout the paper. 


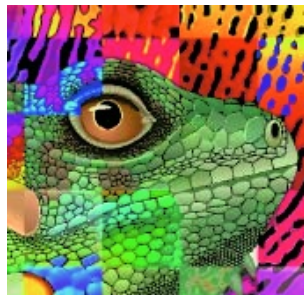

(a)



(b)

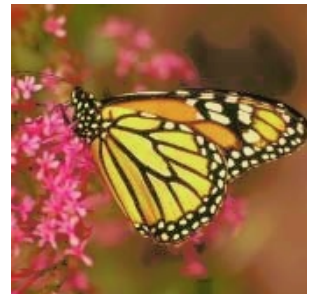

(c)

Fig. 4. Test images: (a) Snake, (b) Girls, (c) Butterfly

Table 1. Obtained objective results

\begin{tabular}{crrrrrrrrrr}
\hline Image & \multicolumn{3}{c}{ Snake } & \multicolumn{3}{c}{ Girls } & \multicolumn{3}{c}{ Butterfly } \\
\hline Method & MAE & MSE & NCD & MAE & MSE & NCD & MAE & MSE & NCD \\
\hline \multirow{2}{*}{ NCS } & 12.446 & 906.1 & 0.2648 & 2.456 & 35.1 & 0.0503 & 3.184 & 70.4 & 0.0449 \\
ACS & 10.729 & 859.4 & 0.2473 & 2.279 & 30.6 & 0.0479 & 2.868 & 59.1 & 0.0420 \\
NSMS & 9.103 & 525.5 & 0.1832 & 1.867 & 16.4 & 0.0420 & 1.768 & 12.8 & 0.0309 \\
ASMS & 7.806 & 460.0 & 0.1590 & 1.742 & 13.8 & 0.0399 & 1.614 & 10.5 & 0.0281 \\
\hline
\end{tabular}

\section{Experimental Results}

To examine the performance of the basic demosaicking solutions designed within the proposed generalized framework, a number of test images have been used. Examples such as the $512 \times 512$ images Snake, Girls, and Butterfly are depicted in Fig.4). These test images, which vary in color appearance and complexity of the structural content (edges), have been captured using three-sensor devices and normalized to 8-bit per channel RGB representation.

Following common practices in the research community [1],[2],[6],[18], mosaic versions of the original color images are created by discarding color information in a GRGR phased Bayer CFA filter (Fig.1a) resulting in the CFA image $z$. The demosaicked images are obtained from applying the demosaicking solution designed within the proposed framework (Fig.3) to process the CFA image. Comparative evaluations are performed by comparing, both objectively and subjectively, the original full color images to demosaicked images. To facilitate the objective comparisons [1], the mean absolute error (MAE), the mean square error (MSE) and the normalized color difference (NCD) criterion are used. While the MAE and MSE criteria are defined in the RGB color space which is conventionally used for storing or visualization purposes, the perceptual similarity between the original and the processed image is quantified using the NCD criterion expressed in the CIE LUV color space [29]. 


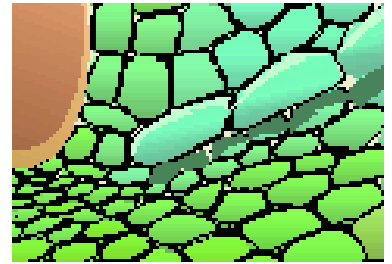

(a1)

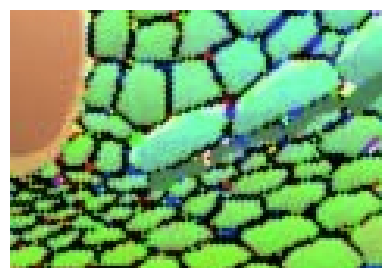

(a2)



(a3)

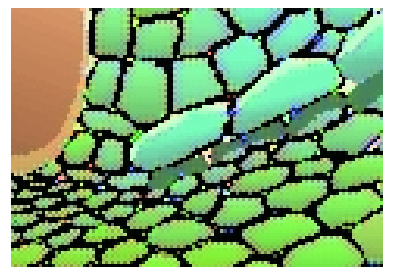

(a4)

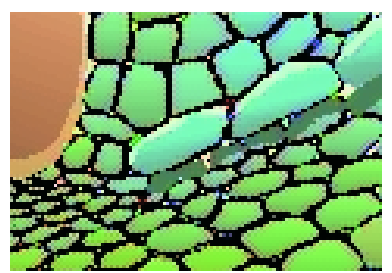

(a5)

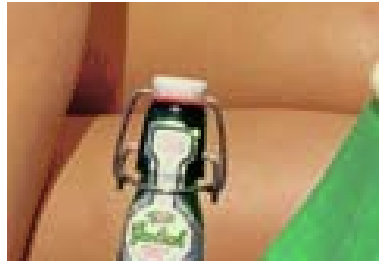

(b1)



(b2)

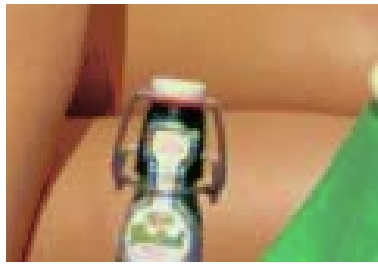

(b3)

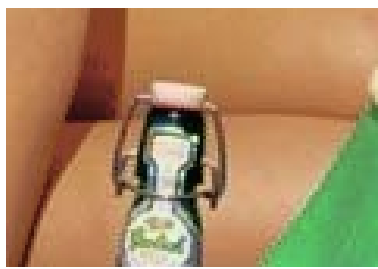

(b4)

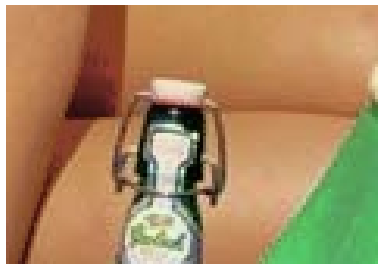

(b5)

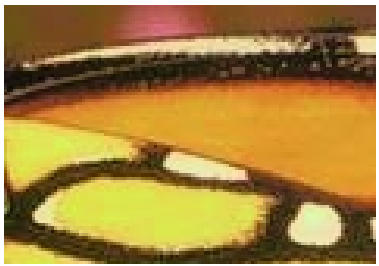

(c1)

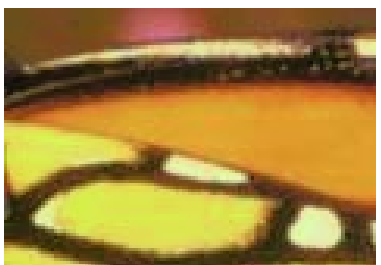

(c2)

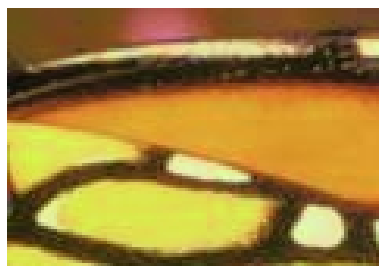

(c3)

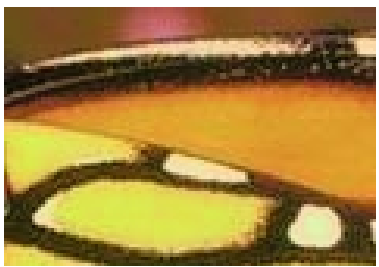

(c4)

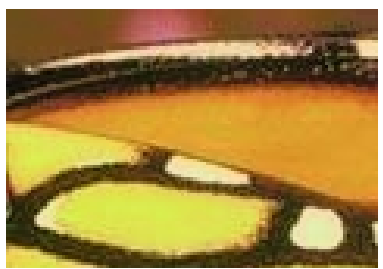

(c5)

Fig. 5. Enlarged parts of the images: (a) Snake, (b) Girls, (c) Butterfly; (1) original image, (2) NCS, (3) ACS, (4) NSMS, (4) ASMS 
To demonstrate the importance of the edge-sensing mechanism and the spectral model, the four solutions designed within the proposed framework (Fig.3) defined in (2),(4), and (5) are considered. Namely, the selected demosaicking schemes include the non-adaptive component-wise scheme (NCS), the adaptive component-wise scheme (ACS), the non-adaptive, spectral model-based scheme (NSMS), and the adaptive, spectral model based scheme (ASMS).

Table 1 summarizes the objective results obtained by comparing the different solutions designed within the proposed demosaicking framework. It can be easily seen that the NCS scheme is the worst performing method among the tested schemes. This should be attributed to its non-adaptive and component-wise nature. The use of the adaptive its adaptive ACS variant improves the result in terms of all objective criteria. However, the significant improvement of the performance of the demosaicking process is observed when the processing solution employs both the spectral model and the edge-sensing mechanism.

Figs. 5 depicts enlarged parts of the test images cropped in edge areas which are usually problematic for Bayer CFA demosaicking schemes. The results show that NCS and ACS solutions blur edges and produce a number of color shifts in the demosaicked image, while the ASMS solution produces the highest visual quality among the tested schemes.

\section{Conclusion}

A generalized demosaicking framework for single-sensor imaging was presented. The framework allows for the utilization of both the spatial and spectral characteristics during the demosaicking process. Experimentation performed here suggests that both the spectral model and the edge-sensing mechanism should be used in the demosaicking pipeline.

\section{References}

1. Lukac, R., Plataniotis, K.N.: Normalized color-ratio modelling for CFA interpolation. IEEE Transactions on Consumer Electronics 50 (2004) 737-745

2. Lukac, R., Plataniotis, K.N., Hatzinakos, D., Aleksic, M.: A novel cost effective demosaicing approach. IEEE Transactions on Consumer Electronics 50 (2004) 256261

3. Ramanath, R., Snyder, W.E., Bilbro, G.L., Sander, W.A.: Demosaicking methods for Bayer color arrays. Journal of Electronic Imaging 11 (2002) 306-315

4. Wu, X., Zhang, N.: Primary-consistent soft-decision color demosaicking for digital cameras. IEEE Transactions on Image Processing 13 (2004) 1263-1274

5. Lukac, R., Martin, K., Plataniotis, K.N.: Digital camera zooming based on unified CFA image processing steps. IEEE Transactions on Consumer Electronics $\mathbf{5 0}$ (2004) 15-24

6. Gunturk, B., Altunbasak, Y., Mersereau, R.: Color plane interpolation using alternating projections. IEEE Transactions on Image Processing 11 (2002) 997-1013

7. Adams, J., Parulski, K., Spaulding, K.: Color processing in digital cameras. IEEE Micro 18 (1998) 20-30 
8. Freeman, W.T.: Median filter for reconstructing missing color samples. U.S. Patent $5373322,(1988)$

9. Cai, C., Yu, T.H., Mitra, S.K.: Saturation-based adaptive inverse gradient interpolation for Bayer pattern images. IEE Proceedings - Vision, Image, Signal Processing 148 (2001) 202-208

10. Lukac, R., Plataniotis, K.N.: Digital camera zooming on the colour filter array. IEE Electronics Letters 39 (2003) 1806-1807

11. Hur, B.S., Kang, M.G.: High definition color interpolation scheme for progressive scan CCD image sensor. IEEE Trans. Consumer Electronics 47 (2001) 179-186

12. Bayer, B.E.: Color imaging array. U.S. Patent 3971065 (1976)

13. Lukac, R., Plataniotis, K.N.: Data-adaptive filters for demosaicking: a framework. IEEE Transactions on Consumer Electronics, submitted (2004)

14. Lukac, R., Plataniotis, K.N., Hatzinakos, D.: Color image zooming on the Bayer pattern. IEEE Transactions on Circuit and Systems for Video Technology 15 (2005)

15. Cok, D.R.: Signal processing method and apparatus for producing interpolated chrominance values in a sampled color image signal. U.S. Patent 4642 678, (1987)

16. Sakamoto, T., Nakanishi, C., Hase, T., Software pixel interpolation for digital still cameras suitable for a 32-bit MCU. IEEE Transactions on Consumer Electronics 44 (1998) 1342-1352

17. Longere, P., Zhang, P., Delahunt, P.B., Brainard, D.H.: Perceptual assessment of demosaicing algorithm performance. Proceedings of the IEEE 90 (2002) 123-132

18. Pei, S.C., Tam, I.K.: Effective color interpolation in CCD color filter arrays using signal correlation. IEEE Trans. Circuits and Systems for Video Technology 13 (2003) 503-513

19. Ramanath, R., Snyder, W.E.: Adaptive demosaicking. Journal of Electronic Imaging 12 (2003) 633-642

20. Kakarala, R., Baharav, Z.: Adaptive demosaicing with the principal vector method. IEEE Transactions on Consumer Electronics 48 (2002) 932-937

21. Hamilton, J.F., Adams, J.E.: Adaptive color plane interpolation in single sensor color electronic camera. U.S. Patent 5629 734, (1997)

22. Kimmel, R.: Demosaicing: image reconstruction from color CCD samples. IEEE Transactions on Image Processing 8 (1999) 1221-1228

23. Lu, W., Tang, Y.P.: Color filter array demosaicking: new method and performance measures. IEEE Transactions on Image Processing 12 (2003) 1194-1210

24. Kehtarnavaz, N., Oh, H.J., Yoo, Y.: Color filter array interpolation using color correlations and directional derivatives. Journal of Electronic Imaging 12 (2003) 621-632

25. Chang, L., Tang, Y.P., Effective use of spatial and spectral correlations for color filter array demosaicking. IEEE Trans. Consumer Electronics 50 (2004) 355-365

26. Lukac, R., Plataniotis, K.N.: A Robust, Cost-Effective Postprocessor for Enhancing Demosaicked Camera Images. Real-Time Imaging, Special Issue on Spectral Imaging II, 11 (2005)

27. Adams, J., Design of practical color filter array interpolation algorithms for digital cameras. Proceedings of the SPIE 3028 (1997) 117-125

28. Lukac, R., Martin, K., Plataniotis, K.N.: Demosaicked image postprocessing using local color ratios. IEEE Transactions on Circuit and Systems for Video Technology 14 (2004) 914-920

29. Plataniotis, K.N., Venetsanopoulos, A.N.: Color Image Processing and Applications. Springer Verlag, Berlin, (2000) 